\title{
¿EVOLUCIONAN LAS EXPORTACIONES COLOMBIANAS HACIA UN MERCADO MÁS VOLÁTIL?: UN ANÁLISIS DEL PERIODO 1974-2014*
}

\author{
JOSEFA RAMONI PERAZZI"* \& GIAMPAOLO ORLANDONI MERLI"* \\ UNIVERSIDAD DE SANTANDER
}

Recibido/ Received/ Recebido: 22/04/2015-Aceptado/ Accepted / Aprovado: 02/08/2016

\begin{abstract}
Resumen
Este estudio busca determinar si la economía colombiana se mueve hacia una estructura de exportaciones más inestable, para lo cual se analiza tanto la evolución de las mismas entre el 1974 y el 2014, como la volatilidad de sus precios; entendida esta como las desviaciones en torno a la media, corregida o no por la tendencia, sin pretender explicar las razones de dicha inestabilidad. Los resultados muestran que las exportaciones colombianas siguen una marcada tendencia a moverse hacia productos minerales y energéticos, antes que agropecuarios. Los precios de los commodities tienden por lo general a crecer, alcanzando sus máximos en la primera década del siglo XXI. Los indicadores de volatilidad considerados, aplicados a los índices de precios de los productos de exportación tradicional y de otros índices de precios combinados, sugieren una mayor volatilidad en los mercados hacia los cuales se mueven las exportaciones de Colombia: los minerales. Con respecto a la tendencia que sigue la volatilidad observada en los precios considerados, los resultados no son concluyentes. Mientras los indicadores básicos apuntan a una creciente fluctuación en los precios agropecuarios, el rango de Parkinson sugiere lo contrario.
\end{abstract}

Palabras clave: Exportaciones; Colombia; Volatilidad; Commodities; Precios del petróleo.

Artículo de investigación, resultado del proyecto de investigación titulado "Comportamiento del mercado de commodities de exportación en Colombia y su impacto en la economía del país", financiado por la Universidad de Santander

* Economista (ULA - Vzla, 1991), Máster en Estadística (ULA - Vzla, 1998) y en Economía (USF - EEUU, 2002), PhD en Economía (USF - EEUU, 2004). Profesora titular jubilada activa del Departamento de Economía de la Facultad de Ciencias Económicas y Sociales de la Universidad de Los Andes (Mérida, Venezuela). Actualmente se desempeña como profesora especial de planta en la Universidad de Santander (Colombia). Pertenece al grupo de investigación Ciempiés (UDES) y Equidad y Bienestar (ULA). Dirección postal: Universidad de Santander, calle 70 \#55-210, Bucaramanga, Santander. Teléfono: 6516500 Ext. 1492. Correo electrónico: j.ramoni@udes.edu.co

Economista (ULA - Vzla, 1971), Máster en Economía e Investigación de Operaciones (ISU - EEUU, 1979), Doctor HC en Estadística (ULA - Vzla, 2013). Profesor titular jubilado activo del Instituto de Estadística Aplicada de la Facultad de Ciencias Económicas y Sociales de la Universidad de Los Andes (Mérida, Venezuela). Actualmente se desempeña como profesor titular de planta en la Universidad de Santander (Colombia). Pertenece al grupo de investigación CIBAS (UDES) y Equidad y Bienestar (ULA). Dirección postal: Universidad de Santander, calle 70 \#55-210, Bucaramanga, Santander. Teléfono: 6516500 Ext.1665. Correo electrónico: gorlandoni@udes.edu.co 


\title{
COLOMBIAN EXPORTS DO THEY EVOLVE TOWARDS A MORE VOLATILE MARKET?: AN ANALYSIS OF THE PERIOD 1974-2014
}

\begin{abstract}
This study seeks to determine whether the Colombian economy moves toward a more unstable export structure, for which analyzes both; the evolution of the economy between 1974 and 2014, price volatility; understood as deviations around the measure, corrected or not by the trend and without trying to explain the reasons for the instability. The results show that Colombian exports follow a marked tendency to move minerals and energy products, rather than agricultural. Commodity prices generally tend to grow, reaching their peak in the first decade of the XXI century. Considered Volatility Indicators applied to traditional export products' price indexes and other combined price indexes suggest greater volatility in the markets to which Colombia exports move: minerals. With regard to the trend following the volatility in the prices considered, the results are inconclusive. While the basic indicators point to a growing fluctuation in agricultural prices, the range of Parkinson suggests otherwise.

Keywords: Exports; Colombia; Volatility; Commodities; Oil prices.
\end{abstract}

\section{AS EXPORTAÇÕES COLOMBIANAS EVOLUEM PARA UM MERCADO MAIS VOLÁTIL?: UMA ANÁLISE DO PERÍODO 1974-2014}

\begin{abstract}
Resumo
Este estudo procura determinar se a economia colombiana se move para uma estrutura de exportações mais instável. Para isto, analisa-se tanto a evolução das mesmas entre 1974 e 2014, como a volatilidade de seus preços, entendida esta como os desvios em torno da média, corrigida ou não pela tendência, sem pretender explicar as razões de dita instabilidade. Os resultados mostram que as exportações colombianas acompanham uma marcada tendência a se mover para produtos minerais e energéticos, mais que agropecuários. Os preços das commodities tendem em geral a crescer, atingindo seus máximos na primeira década do século XXI. Os indicadores considerados de volatilidade, aplicados aos índices de preços dos produtos de exportação tradicional e de outros índices de preços combinados, sugerem uma maior volatilidade nos mercados para os quais se movem as exportações da Colômbia: os minerais. Com relação à tendência que acompanha a volatilidade observada nos preços considerados, os resultados não são concluintes. Enquanto os indicadores básicos apontam a uma crescente flutuação nos preços agropecuários, a faixa de Parkinson sugere o contrário.

Palauras chave: Exportações; Colômbia; Volatilidade; Commodities; Preços do petróleo.
\end{abstract}

Ramoni, J. \& Orlandoni, G. (2017) ¿Evolucionan las exportaciones colombianas hacia un mercado más volátil?: un análisis del periodo 1974-2014. En: Revista de la Facultad de Ciencias Económica: Investigación y Reflexión. rev.fac.cienc.econ, XXV (1). DOI: http://dx. doi.org/10.18359/rfce.2652

JEL: F10, F 14. 


\section{Introducción}

Las exportaciones colombianas se han caracterizado por el predominio de materias primas esenciales o commodities: café, azúcar, petróleo, carbón, ferroníquel, oro, cobre, entre otros. Todos estos productos tienen en común el ser susceptibles de ser comercializados a nivel mundial en su forma natural o producidos, sin posibilidad de diferenciación entre productores, siempre con base en precios fijados en mercados internacionales a partir de las fuerzas de la oferta y la demanda. Se incluyen los productos energéticos (petróleo, carbón, gas, etc.), metales industriales (cobre, zinc, níquel, etc), metales preciosos (oro y plata) y productos agropecuarios (trigo, maíz, soya, café y ganado).

El mercado de commodities está altamente influenciado por expectativas de corto plazo, lo que lo hace comportarse conforme los ciclos económicos y guardar fuerte correlación positiva tanto con la inflación, como con la exposición a eventos naturales $y$ humanos que puedan afectar su oferta y demanda. Se le considera un mercado volátil, con precios influenciados por factores políticos, climáticos, niveles de existencia y crecimiento en demanda, tipo de cambio, restricciones al comercio, reglamentaciones sobre recursos naturales renovables y no renovables, especulación y precios de la energía, ya sea por su efecto directo sobre los costos de producción de commodities en general, como por su incidencia en la demanda y oferta de biocombustibles.

El papel estratégico que los commodities juegan en la seguridad alimentaria y energética de los pueblos, así como su posible impacto en la determinación del tipo de cambio, hace necesario conocer el desempeño futuro de este mercado, más aún para aquellos países que, como Colombia, dependen sustancialmente de su producción y exportación. Sin embargo, la alta volatilidad asociada a los precios de este mercado dificulta esta tarea y se traduce en inestabilidad macroeconómica, pérdida de eficiencia técnica, déficits de balanza de pagos. Las connotaciones de estas fluctuaciones para el país dependerán de la estructura de sus exportaciones, así como de su política cambiaria.
Este estudio tiene un doble propósito. En primer lugar, analizar la evolución de las exportaciones colombianas a fin de determinar que cambios ha habido en la composición de las mismas a lo largo del periodo estudiado, sin abandonar la hegemonía de las materias primas, sin pretender explicar el porqué del comportamiento observado. En segundo lugar, revisar la volatilidad observada de los precios de los principales mercados hacia los cuales éstas se mueven. Los resultados indican que Colombia no ha logrado reducir su dependencia de las exportaciones de materias primas, si bien ha habido algunos cambios al interior de las mismas, con creciente predominio de productos minerales y energéticos, que reemplazan los agrícolas y en los cuales Colombia no es referencia, cuyos efectos perversos se han sentido con mayor fuerza a raíz de la fuerte caída en los precios del petróleo después de una larga y sostenida bonanza. Las exportaciones no tradicionales no se han ampliado, señal de que el país no ha aprovechado las ventajas de los acuerdos comerciales para colocar productos de mayor valor agregado. En materia de precios, los resultados no son claros. Si bien, la mayoría de los indicadores apuntan hacia una mayor volatilidad en los precios del petróleo y metales, antes que en los productos agropecuarios, aquéllos basados en la desviación estándar, corrigiendo o no por tendencia, señalan que la variación experimentada por estas fluctuaciones tiende a ser mayor, y generalmente positivas, en los últimos. Ello contradice la decreciente volatilidad que el rango de Parkinson atribuye a los precios de ese sector.

\section{Evolución de las exportaciones de Colombia}

Hasta el año 2013, las exportaciones colombianas crecieron a un ritmo acelerado, logrando mantener un saldo mayoritariamente positivo en balanza de pagos, no obstante el hecho de que éstas siguen representando entre el 15\% y el 20\% histórico, mientras que las importaciones, si bien se sitúan en el mismo rango, muestran una marcada tendencia a crecer. Este crecimiento se estancó posteriormente, en respuesta tanto al deterioro de los precios del petróleo como a la desaceleración de grandes potencias como China y Estados Unidos. 
Durante los 90's Colombia inicia un proceso de apertura comercial para reemplazar su política altamente proteccionista (la más fuerte entre los países andinos), hacia una economía más abierta, con reducción gradual de barreras arancelarias y no arancelarias, lo que la ha llevado a negociar acuerdos con países en diversos continentes atraídos en su momento, tanto por la bonanza económica que experimentaba el país, como por su estratégica posición geográfica. No obstante ello, su mercado de exportación está dominado cada vez más por productos de exportación tradicionales, café, carbón, petróleo y ferroníquel, todos ellos commodities, lo que la convierte en una economía fuertemente dependiente de precios determinados a nivel internacional (llustración 1) y pone en evidencia la poca capacidad del país para aprovechar las ventajas que la apertura comercial le ofrece para colocar productos de mayor valor agregado.

Algunos rubros han hecho de Colombia un país reconocido a nivel mundial. Uno de ellos es el café, cuya participación en las exportaciones tradicionales ha venido cayendo a niveles alarmantes, pasando de más del $82 \%$ en 1974, a menos del $7 \%$ en el 2014 , espacio que ha sido absorbido paulatinamente por carbón y, principalmente petróleo, tal como se observa en la tabla 1. Aun así, Colombia no es referencia mundial en el mercado de estos dos productos. En efecto, en cuanto a carbón, Colombia exportó en el 2014 aproximadamente 89 millones de toneladas, cerca de 16\% más que en 2013 . El país no figura en la lista de los diez principales productores, encabezados por los Estados Unidos, no obstante ser Australia el principal exportador. En cuanto a petróleo, Colombia produce poco más de un millón de barriles diarios, de los cuales exportó cerca de 500 mil en el 2014; se estima que las reservas actuales de crudo y gas alcanzan para siete y 15 años, respectivamente (Banco de la República, 2014).

Casi el 80\% de los productos que Colombia ofrece al mundo corresponden a materias primas, cuya estructura parece moverse hacia el predominio de productos minerales, en desmedro del agro. En efecto, mientras las exportaciones del primer grupo en promedio crecieron $70 \%$ entre el 2010 y el 2014, las exportaciones de los rubros agrícolas y pecuarios aumentaron apenas 18\% durante el mismo periodo. Por su parte, las exportaciones industriales apenas si lograron crecer 6\%. Dentro de las exportaciones tradicionales, las mayores variaciones desde el 2004 las experimentaron el petróleo (37\%) y el café, (-48\%); el primero, producto en el cual Colombia se abría paso; el segundo, producto

Ilustración 1. Evolución de exportaciones colombianas (en logaritmo)

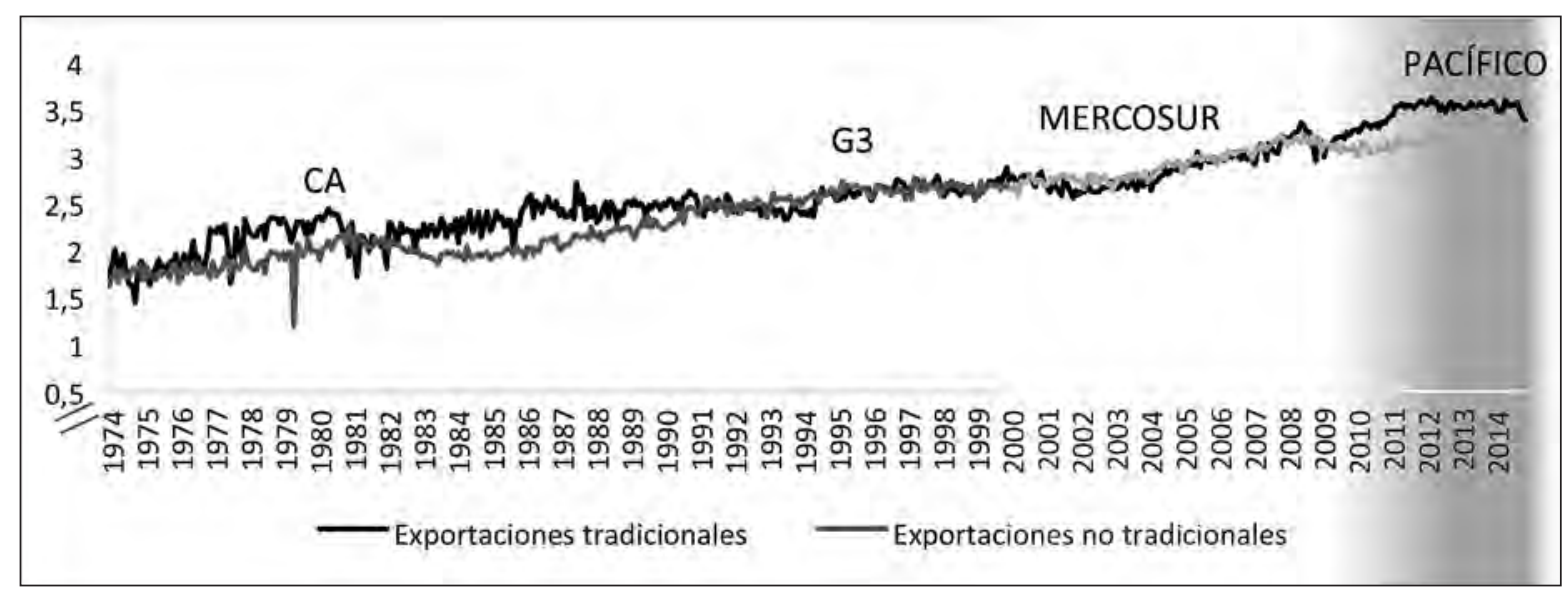

Fuente: DANE. Elaboración propia 
emblemático de ese país, que se vio altamente afectado a raíz de la ruptura en 1989 del Acuerdo Internacional del Café, firmado en 1962 por los países miembros de la Organización Internacional del Café, el cual establecía cuotas de producción que permitían controlar la oferta y garantizar precios estables y razonables.

Tabla 1. Exportaciones tradicionales por rubro (\%)

\begin{tabular}{|c|c|c|c|c|}
\hline Año & Café & Carbón & Petróleo y derivados & Ferroníquel \\
\hline 1974 & 82,9 & 0,4 & 16,7 & - \\
\hline 1984 & 75,3 & 1,6 & 20,5 & 2,7 \\
\hline 1994 & 50,1 & 13,8 & 33,1 & 3,0 \\
\hline 2004 & 12,4 & 24,2 & 55,2 & 8,2 \\
\hline 2013 & 4,5 & 16,0 & 77,8 & 1,6 \\
\hline 2014 & 6,4 & 17,5 & 74,5 & 1,6 \\
\hline
\end{tabular}

Fuente: DANE. Elaboración propia.

Con relación al café, es importante resaltar que, si bien éste ha cedido terreno, en el 2014 mostró un repunte tanto en su producción como en su exportación. Así, durante este último año, la cosecha pasó de 542.820 a 617.959 toneladas. De éstas, el $92 \%$ se destina al mercado internacional, lo que permite suponer que el café seguirá liderando las exportaciones agrícolas, ello sin contar con el efecto positivo en el empleo, por cuanto se estima que cada millón de sacos de café genera 65.000 empleos directos.

Estos auges y caídas han ocurrido en contextos muy variados, lo que ha llevado a varios estudiosos a indagar acerca de los factores determinantes del comportamiento de las exportaciones colombianas. Echevarría (1987) analiza las crecientes exportaciones de la época y concluye que se deben a la dinámica que en su momento imprimió la Asociación Latinoamericana de Libre Comercio (ALALC), mientras que el efecto del tipo de cambio resulta despreciable. Un reciente informe de la Asociación Nacional de Instituciones Financieras (ANIF, 2016) resalta el que las exportaciones colombianas no han tomado ventaja de la devaluación para mejorar su colocación de productos en mercados internacionales. Es que la promoción de exportaciones debe descansar en factores más sólidos.

Bonifaz \& Mortimore (1999) concluyen que Colombia no ha sabido potenciar sus oportunidades de integración a través de mejoras en sectores dinámicos, para lo cual la capacitación del trabajador y la inversión en infraestructura son esenciales. Mesa et al. (2001) enfatiza también el papel que la apertura comercial tuvo en la expansión de las exportaciones colombianas, sin que con ello se generara ningún cambio estructural en las mismas, lo que no garantiza su futura expansión.

Garay (2004) revisa los factores determinantes de la competitividad comercial del país antes y después de la apertura y concluye que no existen factores explicativos sobresalientes. Aun así, el autor resalta que las mejoras salariales eliminaron uno de los principales elementos promotores de las exportaciones de Colombia, las cuales se venían sustentando precisamente en el relativamente bajo costo de la mano de obra. En esa misma línea, Álvarez \& Bermúdez (2012) señalan que la sola ampliación de la frontera de mercado no garantiza la diversificación de la oferta, por cuanto ésta debe venir acompañada con mejoras a la infraestructura y a la productividad de los factores.

Así, todo apunta a que Colombia debe lograr mejoras al interior de su aparato productivo si quiere elevar sus niveles de productividad $y$, por ende, de competitividad, con miras a destacarse en un mercado internacional invadido por países que ofrecen materias primas sin mayor ventaja que sus relativamente bajos costos.

\subsection{Exportaciones no tradicionales}

En el año 2014, las exportaciones no tradicionales representaron menos del 30\% del total, con tendencia a decrecer. Los productos de exportación que las componen se distribuyen en tres sectores básicos: industrial (textiles, químicos, papel y manufacturas, cuero y manufactura y alimentos), agropecuario 
(banano, flores, carnes) y minero (oro, esmeraldas), en orden de importancia (llustración 2).

El peso de cada uno de estos sectores dentro del total de exportaciones no tradicionales no ha variado sustancialmente en cuatro décadas, tal como se evidencia en la siguiente tabla. Aun así, el oro no monetario ha sufrido una disminución en el volumen de exportación por el orden 15\%, según reporta el Banco de la República (2014).

Ilustración 2. Participación de las exportaciones tradicionales y no tradicionales en el total (\%)

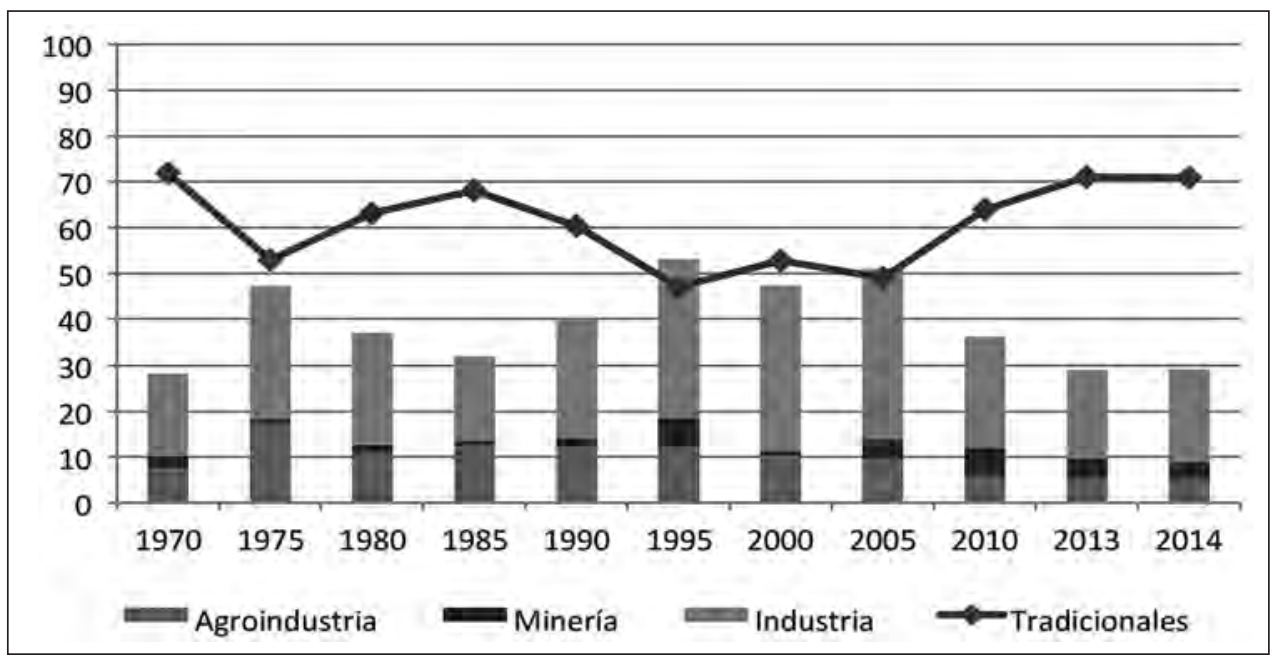

Fuente: DANE. Elaboración propia.

Tabla 2. Exportaciones no tradicionales (\%)

\begin{tabular}{|l|c|c|c|c|c|c|}
\hline \multicolumn{1}{|c|}{ Sector / producto } & $\mathbf{1 9 7 4}$ & $\mathbf{1 9 8 4}$ & $\mathbf{1 9 9 4}$ & $\mathbf{2 0 0 4}$ & $\mathbf{2 0 1 3}$ & $\mathbf{2 0 1 4}$ \\
\hline Agropecuario & $\mathbf{2 4 , 3}$ & $\mathbf{4 2 , 7}$ & $\mathbf{2 7 , 3}$ & $\mathbf{1 7 , 8}$ & $\mathbf{1 7 , 8}$ & $\mathbf{1 7 , 6}$ \\
\hline Banano & - & 42,8 & 3,9 & 26,7 & 25,1 & 29,8 \\
\hline Flores & 9,8 & 28,0 & 34,5 & 43,5 & 43,8 & 49,0 \\
\hline Otros & 90,2 & 29,3 & 26,2 & 29,8 & 31,2 & 21,3 \\
\hline Minero & $\mathbf{3 , 4}$ & $\mathbf{3 , 5}$ & $\mathbf{1 1 , 5}$ & $\mathbf{9 , 2}$ & $\mathbf{1 5 , 5}$ & $\mathbf{1 3 , 0}$ \\
\hline Oro & - & - & 6,8 & 66,9 & 84,1 & 76,1 \\
\hline Esmeraldas & 25,9 & 66,2 & 80,5 & 8,8 & 4,7 & 7,0 \\
\hline Otros & 74,1 & 33,8 & 12,7 & 24,3 & 11,1 & 16,9 \\
\hline Industrial & $\mathbf{7 2 , 2}$ & $\mathbf{5 2 , 8}$ & $\mathbf{6 1 , 3}$ & $\mathbf{7 2 , 9}$ & $\mathbf{6 6 , 7}$ & $\mathbf{6 9 , 4}$ \\
\hline Textiles y manufactura & 30,1 & 17,9 & 26,1 & 17,9 & 8,6 & 7,9 \\
\hline Químicos & 15,9 & 19,3 & 19,6 & 23,9 & 33,5 & 34,0 \\
\hline Papel y manufactura & 2,9 & 12,9 & 7,7 & 7,1 & 4,4 & 3,9 \\
\hline Cuero y manufactura & 4,9 & 5,5 & 8,0 & 3,0 & 2,9 & 3,0 \\
\hline Alimentos & 18,5 & 20,2 & 16,6 & 14,8 & 14,5 & 17,9 \\
\hline Otros & 27,8 & 24,2 & 22,1 & 33,3 & 36,3 & 33,2 \\
\hline
\end{tabular}

Fuente: DANE. Elaboración propia. 


\subsubsection{Sector agropecuario}

El sector agropecuario, que llegó a representar el $42,7 \%$ del total de las exportaciones no tradicionales, cayó en la última década a niveles inferiores a los de 1974. En este sector destacan dos productos, bananos y flores, que han rotado su posición en el total agropecuario. Con respecto a los commodities agrícolas de exportación, diferentes al café, es evidente el predominio del azúcar (29,2\% en 2014), sin clara tendencia. Los restantes rubros tienen muy baja participación en las exportaciones del sector, con tendencia a mantenerse o crecer ligeramente, excepción hecha para el algodón y la madera (tabla 3 ).

\subsubsection{Sector minero}

A nivel de los minerales, las exportaciones de oro y otros minerales han venido reemplazando a las de las esmeraldas, probablemente gracias a los mayores precios del primero. Otros minerales de exportación incluyen el cobre y el aluminio, pero su decreciente participación en el total del sector apenas si supera el 20\% combinado al 2014 (tabla 4).

\subsubsection{Sector industrial}

Este sector representa poco menos del 70\% de las exportaciones no tradicionales, porcentaje que no ha variado en décadas, no obstante los esfuerzos de apertura económica. En este, el desarrollo de una industria, como por ejemplo la de los químicos, pareciera venir a costa del descuido de otra, léase textiles y cueros, lo que probablemente explica su estancamiento. Diversos factores explican el poco dinamismo industrial, citándose entre ellos los elevados costos relativos de la mano de obra y de la energía; su ubicación cercana a las ciudades que sirven y alejada de los puertos, lo que incrementa los de por si elevados costos de transporte y la falta de idoneidad de la infraestructura vial y portuaria.

Tabla 4. Principales commodities de exportación mineral, excluyendo carbón y ferroníquel (\%)

\begin{tabular}{|c|c|c|c|}
\hline Año & Cobre & Aluminio & Plomo \\
\hline 2008 & 19,20 & 13,58 & 0,40 \\
\hline 2009 & 8,78 & 6,52 & 0,16 \\
\hline 2010 & 13,50 & 6,45 & 0,59 \\
\hline 2011 & 11,02 & 4,48 & 0,26 \\
\hline 2012 & 9,21 & 4,63 & 0,19 \\
\hline 2013 & 11,89 & 7,93 & 0,78 \\
\hline 2014 & 12,94 & 9,68 & 0,65 \\
\hline
\end{tabular}

Fuente: DANE. Elaboración propia.

La poca competitividad del sector industrial se ve reflejada en la reducida y decreciente tasa de apertura expor-

Tabla 3. Principales commodities de exportación agrícolas, excluyendo café (\%)

\begin{tabular}{|c|c|c|c|c|c|c|c|}
\hline Año & Azúcar & Algodón & Cacao & Madera & Tabaco & Semillas oleaginosas & Cereales \\
\hline 2008 & 12,42 & 5,09 & 2,40 & 2,17 & 1,17 & 0,44 & 0,21 \\
\hline 2009 & 21,54 & 4,74 & 2,28 & 1,58 & 1,09 & 0,42 & 0,19 \\
\hline 2010 & 28,65 & 3,06 & 3,07 & 1,48 & 0,77 & 0,80 & 0,21 \\
\hline 2011 & 35,01 & 3,89 & 3,10 & 1,32 & 1,21 & 0,88 & 0,16 \\
\hline 2012 & 26,83 & 2,83 & 2,55 & 1,55 & 1,26 & 0,91 & 0,27 \\
\hline 2013 & 21,32 & 1,80 & 3,01 & 1,41 & 2,20 & 0,81 & 0,39 \\
\hline 2014 & 29,20 & 1,57 & 5,20 & 1,94 & 1,93 & 0,95 & 0,78 \\
\hline
\end{tabular}

Fuente: DANE. Elaboración propia. 
tadora $(\mathrm{TAE})^{2},{ }^{1}$ indicativa de cuánto producto colombiano se demanda en el exterior (tabla 5). Este hecho, y el que las exportaciones sigan representando menos del 20\% del PIB, ratifica el rezago del país en el proceso de búsqueda de mercados para productos no tradicionales.

Tabla 5. Tasa de apertura exportadora por sector (\%)

\begin{tabular}{|c|c|c|c|c|c|}
\hline Año & $\begin{array}{c}\text { Cueros } \\
\text { y calzados }\end{array}$ & $\begin{array}{c}\text { Textiles y } \\
\text { confecciones }\end{array}$ & $\begin{array}{c}\text { Papel y } \\
\text { derivados }\end{array}$ & $\begin{array}{c}\text { Madera y } \\
\text { derivados }\end{array}$ & Alimentos \\
\hline 2001 & 29,38 & 32,49 & 15,61 & 28,01 & 10,59 \\
\hline 2005 & 31,81 & 39,74 & 17,36 & 21,08 & 12,45 \\
\hline 2010 & 26,42 & 27,85 & 19,68 & 9,97 & 8,46 \\
\hline $2013^{*}$ & 27,24 & 25,06 & 21,39 & 13,91 & 8,96 \\
\hline
\end{tabular}

* Última cifra disponible

Fuente: DANE. Elaboración propia.

\subsection{Análisis conjunto}

El Análisis de Componentes Principales (ACP) brinda una idea más clara de la evolución histórica de la estructura de las exportaciones colombianas. El $\mathrm{ACP}$ es una técnica estadística de síntesis que permite reducir la dimensionalidad de las variables, al construir componentes o factores como combinaciones lineales de las variables originales, con base en las correlaciones que existen entre ellas. Esos factores deben contener una alta proporción de la información contenida en el conjunto de variables originales. Así, es posible conocer su estructura interna y la relación, a veces no tan evidente, que existe entre las variables objeto de estudio.

Los componentes obtenidos permiten establecer relaciones entre las variables originales, mostrando de qué manera se asocian o cómo se distancian entre ellas. La asociación entre las variables originales y los componentes se cuantifica mediante las cargas (loadings), los indican la importancia de cada variable en la construcción del respectivo componente. Para su interpretación, se debe tomar en cuenta el valor absoluto de las cargas de cada variable dentro del componente, distinguiendo entre las que tienen valores grandes y valores pequeños. A mayor valor,

\footnotetext{
TAE=Exportación / Producción industrial
}

mayor peso de la variable. Seguidamente, se analiza el signo de las cargas mayores: signos iguales indican que las variables se mueven en la misma dirección; signos diferentes, sugieren que las variables se mueven en sentidos opuestos. La interpretación se hace a partir de los grupos de variables con signo positivo versus grupos con signo negativo. La bondad de esta metodología es que permite una representación gráfica de las cargas, lo que facilita su interpretación.

Utilizando información del porcentaje de cada uno de los trece principales productos de exportación colombianos dentro del total durante el periodo 19712014 , se obtienen cuatro componentes con valores propios mayores que uno, cuyo poder explicativo de la variabilidad total suma $84,47 \%$. Para efectos prácticos, se muestran los resultados gráficos de los dos principales. El primero de ellos (horizontal), explica el $37,93 \%$ de la variabilidad total de los datos. Este componente se define como cafetero-minero, por cuanto contrapone las exportaciones de café (CAF) con las exportaciones minerales que incluyen petróleo (PET), carbón (CAR), ferroníquel (FER). A estos se agregan químicos (QUI), papas (PAP) y flores (FLO). Ello sugiere que el predominio de las exportaciones minerales implica un abandono del café (Ilustración 3a).

El segundo componente, con un poder explicativo del 26,92\%, contrapone exportaciones minerales con las de industrias emblemáticas como lo son los textiles (TEX) y cueros (CUE) y sus derivados. Ello de nuevo sugiere que la mayor producción para exportación mineral se asocia con un menor peso de la industria textil y del cuero. Los valores de estas cargas y las de los restantes componentes se aprecian con detalle en el tabla 6.

Si en el plano generado por estos dos componentes se sobreponen los años considerados en el estudio, se obtiene un mapa bastante gráfico de la evolución de las exportaciones colombianas. En efecto, en los años 1970s el café aparece como producto predominante, que fue cediendo paso a productos con un cierto componente industrial, como son alimentos (1980s) y papel y sus derivados, conjuntamente con las flores (1990s y 2000s), para finalmente exhibir un marcado predominio del carbón y del petróleo en los años recientes (Ilustración 3b). 
Ilustración 3. Análisis de componentes principales sobre las exportaciones

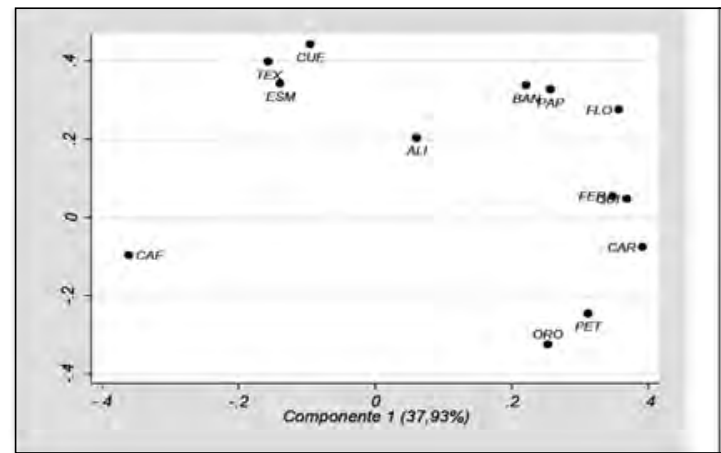

a) Gráfico de las cargas de los factores

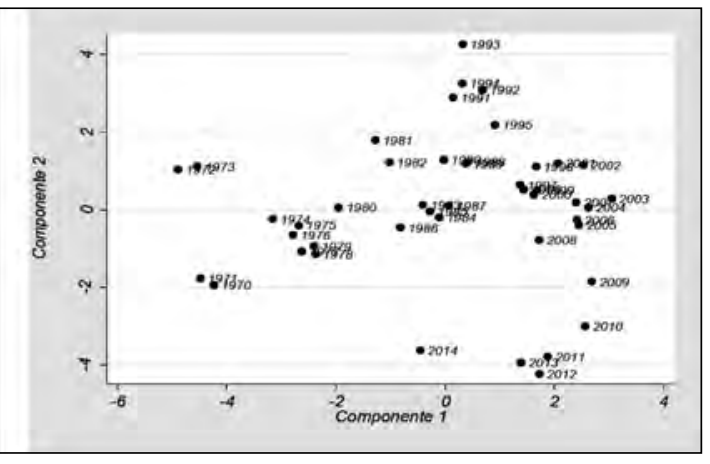

b) Gráfico de las observaciones (años)

Fuente: DANE. Elaboración propia en STATA 12.

Tabla 6. Cargas de los componentes principales

\begin{tabular}{|c|c|c|c|c|}
\hline Variable & Comp1 & Comp2 & Comp3 & Comp4 \\
\hline CAF & $-0,361$ & $-0,0969$ & 0,308 & 0,025 \\
\hline CAR & 0,392 & $-0,0758$ & 0,157 & 0,286 \\
\hline PET & 0,311 & $-0,2456$ & 0,134 & 0,345 \\
\hline FER & 0,348 & 0,0539 & 0,055 & 0,131 \\
\hline BAN & 0,221 & 0,3388 & $-0,305$ & 0,070 \\
\hline FLO & 0,357 & 0,2755 & $-0,110$ & $-0,154$ \\
\hline OR0 & 0,253 & $-0,3257$ & 0,212 & 0,269 \\
\hline ESM & $-0,139$ & 0,3437 & 0,201 & 0,462 \\
\hline TEX & $-0,156$ & 0,3989 & 0,345 & 0,116 \\
\hline PAP & 0,368 & 0,0474 & 0,354 & $-0,187$ \\
\hline QUI & 0,257 & 0,3278 & $-0,184$ & $-0,268$ \\
\hline CUE & $-0,095$ & 0,4431 & $-0,036$ & 0,420 \\
\hline ALI & 0,060 & 0,2041 & 0,624 & $-0,414$ \\
\hline
\end{tabular}

Fuente: DANE. Elaboración propia.

Se concluye que la economía colombiana ha venido moviéndose hacia una estructura de exportación alejada de los rubros agrícolas a favor de los minerales, sin abandonar su dependencia de los commodities, no obstante una inicial transición hacia productos industriales. Llama también la atención que los rubros textiles y cueros no aparecen en el mapa como pre- dominantes en periodo alguno, no obstante ser considerados como sectores potenciales en Colombia. Así, el inicialmente creciente sector manufacturero pareciera haber cedido ante el peso de las exportaciones de combustible, mientras que las de metales y minerales, incluyendo oro y plata, tiene bajo peso relativo, según se observa en la Ilustración 4 .

Una mirada al destino de las exportaciones colombianas explica en parte el porqué de estos cambios al interior de una rígida estructura externa sustentada en commodities. Estados Unidos ha sido y sigue siendo el principal comprador, a pesar de una sensible caída en el total de exportaciones hacia ese pais, debida a la recesion económica y a la menor demanda de carbón por parte de ese país, producto de regulaciones internas. La canasta de productos colombianos que adquiere está constituida básicamente por bienes primarios: carbón, petróleo, café, flores y oro. La debilitada economía venezolana ha hecho que ese país deje de ser el segundo principal receptor de exportaciones de Colombia, espacio que se ha diluido entre otros países de la región.

La Unión Europea no ha variado sustancialmente su captación de productos colombianos, mientras que China ha ido ganando terreno, pero siempre con importaciones minerales. Así, los socios comerciales siguen siendo los mismos, con predominio de 
países vecinos quienes, a su vez, siguen demandando el mismo tipo de bienes (Ilustración 5).

Como se indicó anteriormente, el estímulo a las exportaciones no tradicionales requiere tanto de nuevos mercados, como de entrar a competir con productos con mayor valor agregado. Por ahora, Colombia depende de productos de exportación, no renovables, con características muy particulares. Uno, el carbón, tiene una demanda y precio, altos pero en descenso, producto de sustitución por combustibles menos contaminantes y por la incorporación de Estados Unidos al mercado exportador del mismo. Para el segundo, el petróleo, Colombia parece no contar con reservas suficientes, pero tampoco parece haber podido aprovechar en su totalidad los altos precios que tuvo este rubro hasta el 2013, para rescatar y cimentar las bases de una industria alternativa que cuente con la infraestructura que la inserción del país en el mercado internacional requiere.

Ilustración 4. Exportaciones de mercaderías (\%)

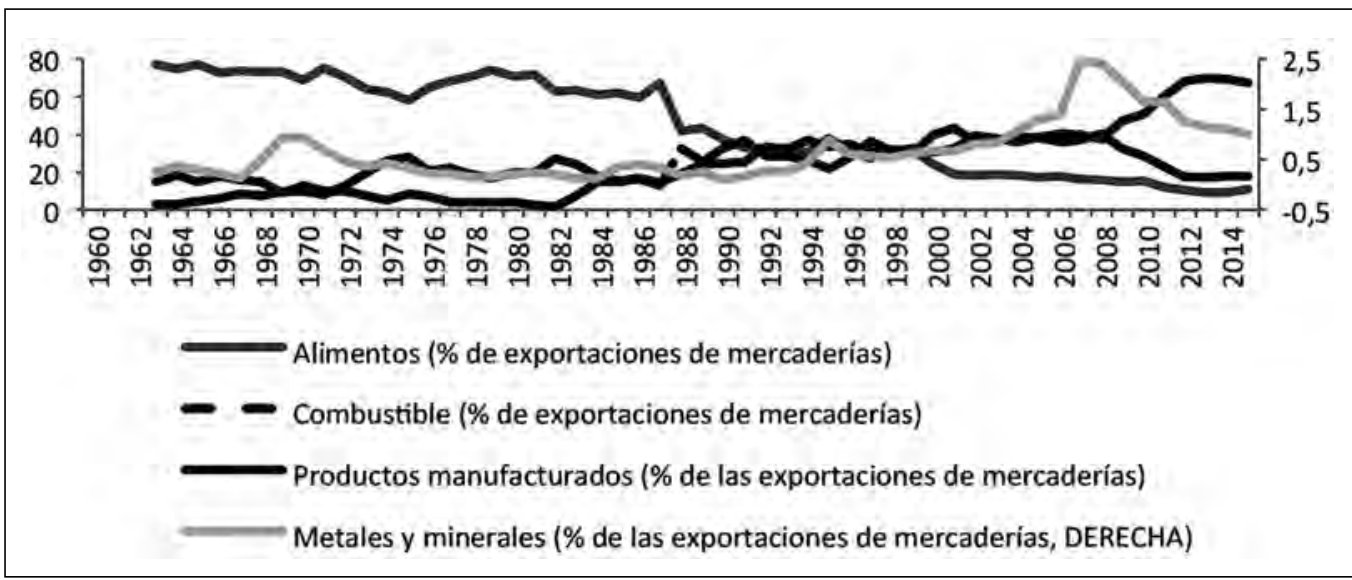

Fuente: Banco Mundial. Cálculos propios.

Ilustración 5. Destino de las exportaciones colombianas

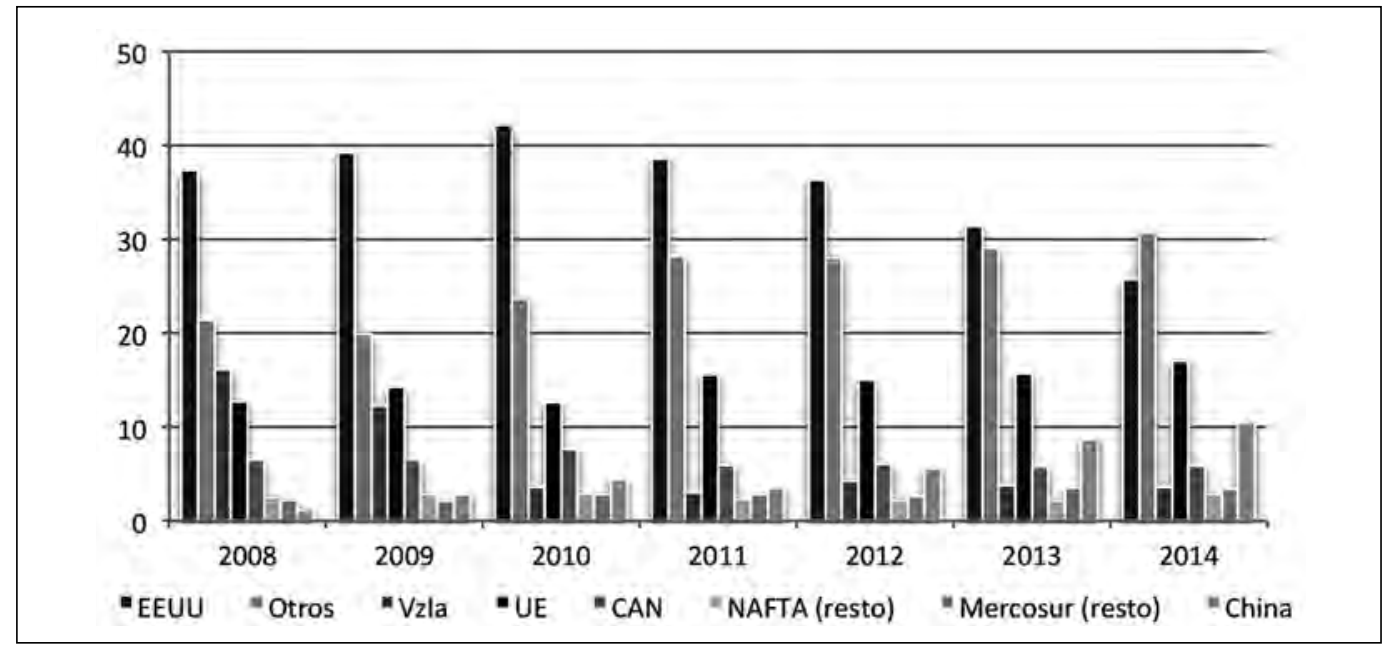

Fuente: DANE. Elaboración propia. 


\section{Precios de los commodities de expor- taciones y eficiencia de los mercados}

Como se indicó en la sección anterior, la expansión de las exportaciones colombianas se atribuye principalmente al petróleo y al carbón, materias primas que no representan mejores oportunidades en los acuerdos de comercio internacional. Ambos productos alcanzaron altos precios en los últimos años, compensando uno las caídas de precio del otro. Visto así, como en anteriores ocasiones, el boom de exportaciones es temporal y su dinámica no depende de Colombia, por tratarse de commodities, cuyo precio se fija a nivel internacional. Varios estudios han intentado analizar la dinámica de los precios de las materias primas.

Gilbert (2003) estudia la evolución de los precios individuales de 21 commodities agrícolas durante el periodo 1960-2002, prestando atención tanto a su tendencia como a su volatilidad. Con respecto a la tendencia, el estudio concluye que existe una marcada caída en los precios, debida probablemente a especulación y la creciente productividad, que permite mayores cosechas. Después de aplicar diversas pruebas de raíz unitaria, no logra determinar si esta tendencia es determinística o estocástica. La volatilidad la analiza a partir de la desviación estándar intra-anual de los cambios en el logaritmo de los precios deflactados y del rango inter-anual escalado de Parkinson (1980).

Gilbert (2003) concluye que la volatilidad ha ido reduciéndose, aun cuando el comportamiento no es similar para todos los rubros considerados. Años más tarde, Gilbert \& Morgan (2010) utilizando, entre otros, modelos generalizados autoregresivos consistentemente heterocedásticos, ratifican la idea de que los mercados agrícolas no han aumentado su volatilidad, y que cada periodo de alta fluctuación es seguido por periodos de estabilidad, lo que coincide con los resultados de Balcombe (2009) y Sumner (2009).

Un estudio más reciente, llega a conclusiones diferentes. Según señala la CEPAL-FAO-IICA (2011) el precio de los commodities agrícolas ha venido incrementándose en los últimos años, luego de haber experimentado una tendencia decreciente. Igualmente, los precios del petróleo han comenzado a retroceder después de haber alcanzado los niveles más altos y por el periodo más prolongado en su historia. Los precios de las materias primas en general tienden a ser más volátiles que los precios de los bienes manufacturados (Jacks, O'Rourke \& Williamson, 2009). Utilizando datos de 30 commodities energéticos y no energéticos durante más de 160 años, Jacks (2013) confirma que estos precios vienen creciendo de manera consistente desde 1950.

Huchet-Bourdon (2011) analiza la evolución de los precios de commodities agrícolas, con énfasis en el periodo 2006-2009, y el impacto que sobre éstos tienen la tasa de cambio y los precios del petróleo y fertilizantes. Para el estudio de la volatilidad, hace uso de coeficiente de variación, ajustado o no por coeficiente de determinación, así como la desviación estándar de la diferencia de los logaritmos, todo ello aplicados a promedios móviles de las series estudiadas. Con respecto a las relaciones entre variables, la matriz de correlaciones y los test de causalidad de Granger muestran que la correlación entre precios de productos agrícolas es más fuerte a medida que los precios aumentan y cambian con la periodicidad de las series. Sus resultados muestran que el impacto de los precios de insumos energéticos y fertilizantes sobre los precios de los productos agrícolas varía según la década, siendo mayor durante los años 2000s, mientras que el tipo de cambio no tiene un efecto significativo sobre los mismos.

En esta primera fase del estudio, se busca identificar cuáles de estos rubros o grupos de bienes muestran mayor volatilidad observada, entendida ésta como la variabilidad histórica exhibida alrededor de la media en las series de tiempo. En este sentido, el estudio ignora la volatilidad implícita, o incertidumbre acerca del comportamiento futuro del mercado, mas asociado a la noción de riesgo y cuya metodología de análisis requiere modelos estadísticos más avanzados, resumidos en Labys (2006). Para ello, se recurre a los siguientes indicadores de uso común en el análisis de volatilidad sin recurrir a modelos complejos. Antes que la comparación de los valores de los indicadores entre sí, nos interesa comparar los resultados obtenidos a partir de ellos para las distintas series analizadas, buscando consistencia en las conclusiones: 
- Coeficiente de variación (CV), dado por el cociente entre la desviación estándar (S) de una variable y su media $(\bar{y})$, de modo que la variabilidad se mide como porcentaje de desviación en torno a la media, lo que resulta apropiado en caso variables con esperanzas matemáticas diferentes:

$$
C V=\frac{S y}{\bar{y}}
$$

- Coeficiente de variación ampliado (CVA) o corregido por el coeficiente de determinación $\left(\mathrm{R}^{2}\right)$ de la regresión de la variable contra el tiempo. De este modo, el tradicional CV se pondera por la desviación estándar residual, una vez controlada la tendencia de la variable en el tiempo:

$$
C V A=C V^{*} C V \sqrt{\left(1-R^{2}\right)}
$$

- Desviación estándar de la tasa de variación (DEV), la cual también permite corregir la posible volatilidad producto del comportamiento tendencial de las series [Gilbert (2006), Jakcs, et al. (2009), Gilbert y Morgan (2010)]:

$$
D E V=D E\left(\ln \frac{y_{t}}{y_{t-1}}\right)
$$

- Rango inter-anual escalonado de Parkinson (REP): que se obtiene a partir de la diferencia entre el logaritmo (ln) de los valores máximo y mínimo de la serie

$$
R E P=\frac{\ln (\max y)-\ln (\min y)}{2 \sqrt{\mathrm{h} 2}}
$$

Donde $(\max y)$ y $(\min y)$ se refieren al mayor y menor valor de la serie en el año, respectivamente. La literatura sugiere que el rango de Parkinson es más robusto que el coeficiente de variación aplicado a datos deflactados. Para el caso de las series consideradas en este estudio, no se hace necesario deflactar, por cuanto se trata de índices de precios los cuales, por naturaleza, se encuentran en términos reales. Estos indicadores se aplican a índices mensuales de precios internacionales de los principales rubros de exportación del país (café, petróleo, carbón y ferroníquel) para el periodo 1981:01-2014:07, de manera individual, así como a diversos índices combinados de precios mensuales agropecuarios y minerales ${ }^{3},{ }^{2}$ todos los cuales incluyen productos de exportación no tradicionales colombianos, tal como se detalla en la tabla 7.

Al analizar la Ilustración 6 se observan patrones similares en el comportamiento de los precios de ambos sectores, con mayores fluctuaciones sobre todo a partir de la década de los 2000s, con una marcada tendencia a crecer desde los primeros años de la primera década del siglo XXI. En el caso de los rubros agropecuarios, esta tendencia creciente se revierte a partir del 2011, aun cuando lo hace de manera errática. En el caso del petróleo y minerales considerados en el estudio, los máximos valores se observan en el 2007 y 2008 (ferroníquel, petróleo y CRBMetal) y en el 2011 para las restantes variables. Cabe destacar la importante caída que registran los precios del petróleo, después del alza más alta y sostenida en la historia de los precios de este rubro.

Siguiendo a Huchet-Bourdon (2011), todos los indicadores antes mencionados se aplican no sólo a las series originales, sino que se calculan también a partir de series suavizadas a través de promedio móvil centrado con una ventana de doce meses, a fin de corregir fluctuaciones estacionales. Los resultados de los indicadores antes mencionados sugieren que efectivamente existe una relativamente menor volatilidad en el comportamiento general de los precios del sector agropecuario para todo el periodo considerado, aun cuando los resultados no son consistentes. Así, por ejemplo, los precios del petróleo y del ferroníquel son los que mayor volatilidad muestran, tanto a nivel (CV y CVA) y rango (REP) como el términos de su tasa de variación (DEV). Le sigue el carbón, aun cuando su DEV y REP son menores que los del precio del café. PMETAL tienen una alta volatilidad, según el coeficiente de variación, mientras que el CRB-Metal muestra fluctuaciones menores a las del precio del café. Por

Los datos fueron obtenidos a través del Fondo Monetario Internacional y de Bloomberg LP Limited Partnership, gracias a los servicios del Punto de Bolsa de la Universidad de Santander, Colombia. 
Tabla 7. Resumen de Índices de precios compuestos, utilizados en el estudio

\begin{tabular}{|c|c|c|c|c|}
\hline Sector & Nombre & Referencia & Rubros & Periodo \\
\hline \multirow{5}{*}{ 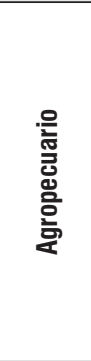 } & GSC-Agro & $\begin{array}{l}\text { Goldman Sachs Commodity Index de bienes agrí- } \\
\text { colas de Standard \& Pool }\end{array}$ & Trigo, maíz, soya, algodón, azúcar, café y cacao & $\begin{array}{r}2009: 08 \\
\text { a 2014:12 }\end{array}$ \\
\hline & PFood & Fondo Monetario Internacional & $\begin{array}{l}\text { Cereales, aceites vegetales, carnes, pescados y ma- } \\
\text { riscos, azúcar, bananas y naranjas }\end{array}$ & $\begin{array}{l}1991: 01 \\
\text { a 2014:12 }\end{array}$ \\
\hline & PFandB & Fondo Monetario Internacional & $\begin{array}{l}\text { Todos los rubros incluidos en PFood, mas café, ca- } \\
\text { cao y te }\end{array}$ & $\begin{array}{r}1991: 01 \\
\text { a 2014:12 } \\
\end{array}$ \\
\hline & CRB-Food & $\begin{array}{l}\text { Commodity Research Bureau Index del Bureau of } \\
\text { Labor Statistics de los Estados Unidos }\end{array}$ & $\begin{array}{l}\text { Carnes, manteca, mantequilla, aceite de soya, cacao, } \\
\text { maíz, trigo, azúcar }\end{array}$ & $\begin{array}{r}1983: 05 \\
\text { a 2014:12 } \\
\end{array}$ \\
\hline & PRAW & Fondo Monetario Internacional & Madera, algodón, lana, caucho y cueros & $\begin{array}{r}1980: 01 \\
\text { a 2014:12 }\end{array}$ \\
\hline \multirow{2}{*}{ 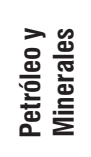 } & PMETAL & Fondo Monetario Internacional & $\begin{array}{l}\text { Cobre, aluminio, hierro, estaño, níquel, zinc, plomo, } \\
\text { y uranio }\end{array}$ & $\begin{array}{c}1980: 01 \\
\text { a } 2014: 12\end{array}$ \\
\hline & CRB-Metal & $\begin{array}{l}\text { Commodity Research Bureau Index del Bureau of } \\
\text { Labor Statistics de los Estados Unidos }\end{array}$ & Cobre, plomo, acero, zinc, estaño & $\begin{array}{l}1994: 01 \\
\text { a 2014:12 }\end{array}$ \\
\hline
\end{tabular}

Fuente: Elaboración propia.

Ilustración 6. Evolución de precios por rubro y sector considerado

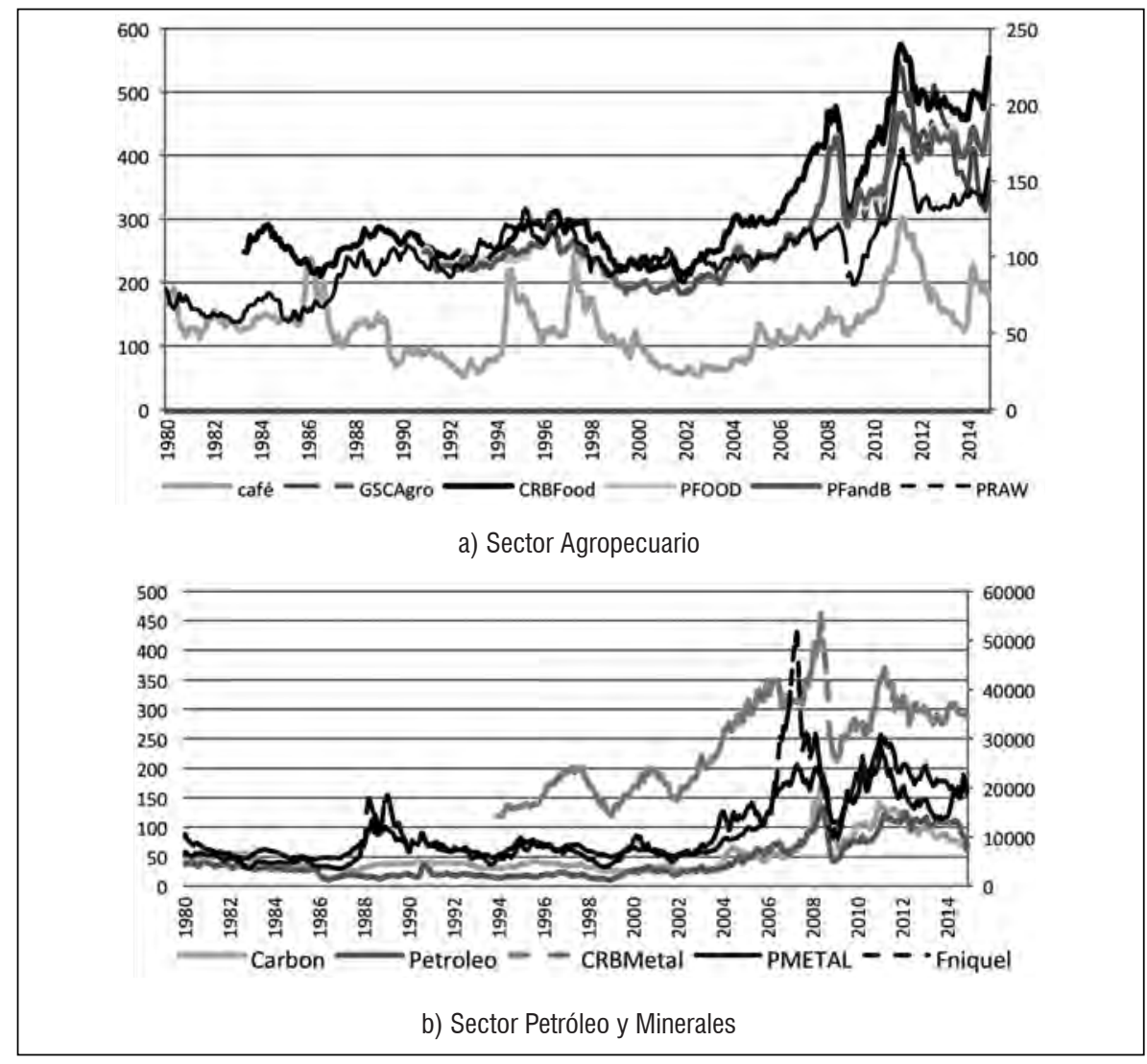

Fuente: Bloomberg y FMI. Elaboración propia. 
lo general, todos los índices que incluyen productos de origen animal y vegetal son menos volátiles que los que se refieren a rubros de origen mineral, aun cuando el café, por sí sólo, muestra altos niveles de volatilidad (tabla 8) ${ }^{4} .{ }^{3} \mathrm{Al}$ trabajar con series suavizadas, los valores de los indicadores basados en el coeficiente de variación (identificados con el subíndice MA) se desinflan, ubicándose por debajo de los originales, pero mostrando el mismo patrón de comportamiento que los originales. Los valores del REP aumentan, pero siguen apuntando hacia una mayor volatilidad en el mercado minero. Sólo el DEV no muestra un patrón claro de comportamiento, con valores por encima o por debajo de los obtenidos para la serie original. Aun así, se evidencia menor volatilidad relativa en los rubros agrícolas.

Siendo que la Ilustración 6 sugiere que las variables exhiben un comportamiento diferenciado a partir de la década 2010 s, se calcula la variación porcentual que experimentan los indicadores, para cada variable, a partir del 2010, con respecto a décadas anteriores. Sorprende ver cómo las mayores diferencias de comportamiento entre ambos periodos corresponden a las variables del sector agropecuario, en especial CRB-Food. Sin embargo, una vez más los resultados son poco claros, tanto en términos de la magnitud de la variación, como en su dirección. El Rango de Parkinson, calculado por décadas, sugiere que la volatilidad del café ha decaído, en concordancia con el comportamiento de CVA y DEV (Ilustración 7).

En la evolución del REP para el promedio de los índices de precios del sector agropecuario (AGRO), se observa una muy baja y relativamente estable volatilidad, mientras que petróleo, carbón y ferroníquel, así como el promedio de los índices de precios de metales (MINERAL) muestran mayores y crecien-

3 La evaluación de la volatilidad con base en el CV y el CVA para estas series, una vez corregida la no estacionariedad con primera diferencia, permite llegar a conclusiones similares, aun cuando los valores difieran de los obtenidos usando los datos originales. En efecto, en este caso para rubros agrícola el CV van de 25,14 (café) a 11,18 (CRB-Food), mientras que para rubros minerales el mayor valor lo muestra petróleo $(37,85)$ y el menor CRB-Metal $(27,70)$. Siendo que no es posible obtener todos los indicadores para series en diferencia, no se reportan los resultados. tes fluctuaciones. Estos resultados parecen apuntar hacia una decreciente volatilidad en el sector agropecuario y una persistente volatilidad en los rubros energéticos y minerales en general.

\section{Conclusiones}

No cabe duda de que la economía colombiana vivió hasta el 2013 su mejor época. Los cuantiosos recursos que llegaron al país habrían podido apalancar tanto el desarrollo de la infraestructura que el país requiere para acelerar su expansión económica, como promover la participación de sectores de producción no tradicionales, con mayor valor agregado. La apertura económica y alianzas con socios estratégicos son prueba de ello. Aun así, Colombia mantiene su alta dependencia de exportaciones de materias primas esenciales, lo que pone de manifiesto el desaprovechamiento que las circunstancias económicas y políticas le están ofreciendo.

Así como el peso de las exportaciones en el total del producto nacional no ha variado significativamente en las últimas décadas, tampoco su composición, en términos gruesos, si bien algunos rubros han sido desplazados por otros. Es por ello que después de varias décadas y luego de varios acuerdos internacionales, las exportaciones tradicionales de café, carbón, petróleo y ferroníquel siguen representando más de las tres cuartas partes del total, con el petróleo ocupando cada vez mayores espacios, no obstante la contracción actual de sus precios.

La industria mantiene un bajo perfil, sin que la tasa de apertura exportadora haya mostrado incrementos significativos. Sobre este particular cabe señalar que a raíz del auge petrolero, Colombia dio síntomas típicos de lo que se conoce como la enfermedad holandesa, no obstante este bajo perfil. En efecto, la mayor entrada de divisas por los elevados precios del crudo vino de la mano del descuido en la producción de otros rubros tradicionales y de los no tradicionales. La caída de los precios del petróleo a partir del 2013 revirtió este proceso, no sin consecuencias sobre el tipo de cambio y el gasto público.

Cabe destacar, que durante los años 80s Colombia ya había vivido una situación similar aunque con me- 
Tabla 8. Indicadores de volatilidad

\begin{tabular}{|c|c|c|c|c|c|c|c|c|c|c|c|}
\hline Indice & CV & CVMA & Variacion(\%) & CVA & CVAMA & Variacion(\%) & DEV & DEVMA & Variacion(\%) & REP & REPMA \\
\hline Café & 0,383 & 0,356 & 45,72 & 0,380 & 0,352 & $-12,00$ & 0,078 & 0,252 & $-23,75$ & 0,208 & 0,381 \\
\hline GSC-Agro & 0,173 & 0,117 & - & 0,173 & 0,118 & - & 0,055 & 0,025 & - & 0,165 & 0,168 \\
\hline PFO0D & 0,295 & 0,282 & 172,96 & 0,196 & 0,187 & 9,65 & 0,029 & 0,010 & 44,66 & 0,083 & 0,193 \\
\hline PFANDB & 0,295 & 0,282 & 172,41 & 0,195 & 0,187 & 0,55 & 0,028 & 0,277 & 48,05 & 0,081 & 0,193 \\
\hline CRB-Food & 0,299 & 0,285 & 190,91 & 0,202 & 0,193 & 168,53 & 0,026 & 0,010 & 57,69 & 0,073 & 0,199 \\
\hline PRAW & 0,234 & 0,220 & $-15,95$ & 0,146 & 0,137 & $-21,72$ & 0,032 & 0,011 & 1,34 & 0,093 & 0,144 \\
\hline Carbón & 0,585 & 0,570 & 138,80 & 0,464 & 0,452 & 91,75 & 0,053 & 0,188 & 107,11 & 0,133 & 0,468 \\
\hline Petróleo & 0,769 & 0,758 & 44,13 & 0,546 & 0,538 & $-22,28$ & 0,086 & 0,189 & $-2,06$ & 0,208 & 0,549 \\
\hline Ferroniquel & 0,706 & 0,680 & 23,06 & 0,544 & 0,524 & 15,26 & 0,084 & 0,438 & 16,88 & 0,253 & 0,548 \\
\hline PMETAL & 0,577 & 0,574 & 116,68 & 0,389 & 0,386 & 27,00 & 0,046 & 0,059 & 23,80 & 0,140 & 0,390 \\
\hline CRB-Metal & 0,324 & 0,307 & 38,69 & 0,260 & 0,247 & 27,76 & 0,047 & 0,058 & 32,40 & 0,132 & 0,263 \\
\hline
\end{tabular}

Fuente: Bloomberg, FMI. Cálculos propios.

Ilustración 7. Rango inter-anual escalonado de Parkinson

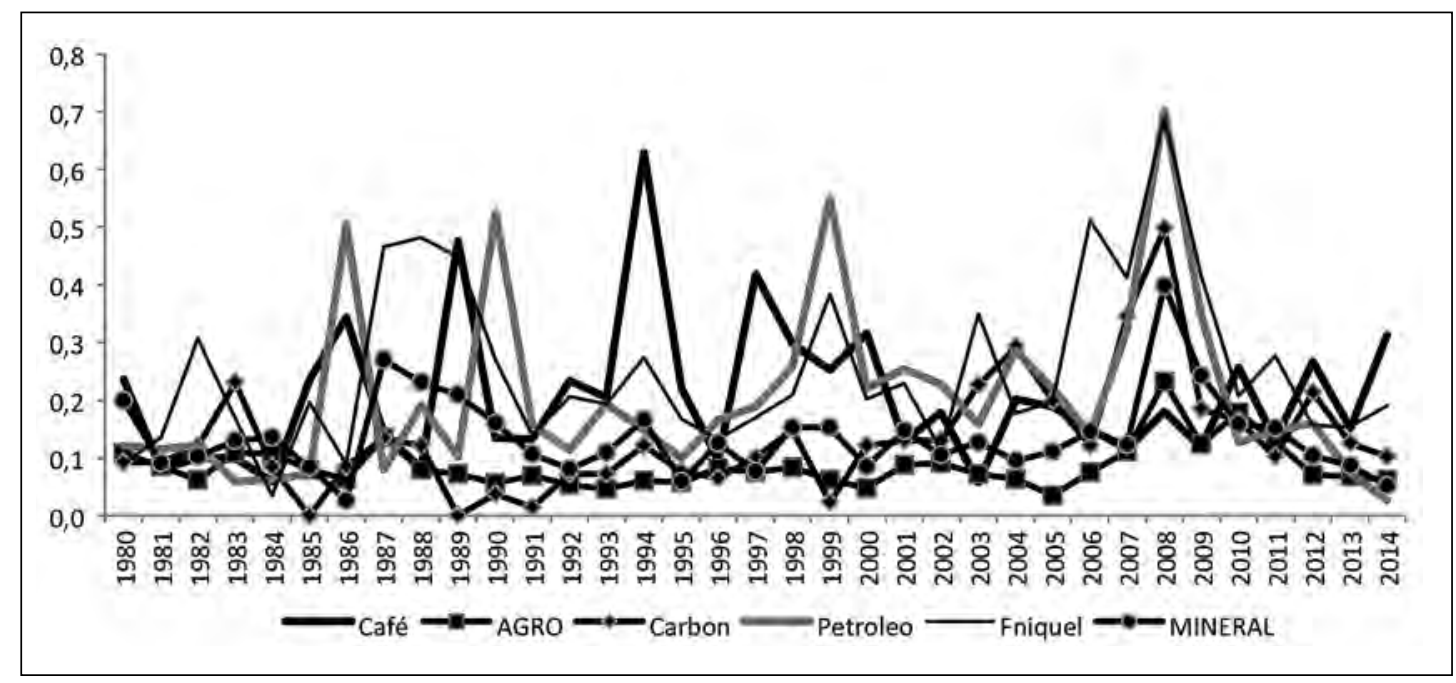

Fuente: Bloomberg, FMI. Elaboración propia.

nor intensidad, a raíz del aumento de los precios del café.

Aun así, el análisis de la evolución de las exportaciones colombianas no deja lugar a dudas en cuanto a su orientación: luego de ser una economía basada en el café, pasando por un periodo de incipiente desarrollo de algunos sectores industriales (papel, textil, cueros, etc.), las exportaciones colombianas de los últimos años han estado dominadas por el petróleo y el carbón, ambos productos sobre cuyos precios Colombia no ejerce ningún control y cuya producción, o bien es limitada (petróleo), o bien tiende a decrecer a nivel internacional (carbón). Se 
concluye, entonces, que las exportaciones de Colombia se mueven hacia productos minerales, en detrimento de los agrícolas y pecuarios.

A nivel mundial, existe una creciente preocupación por la volatilidad exhibida por los precios de las materias primas esenciales o commodities, en particular los agrícolas y los energéticos. Ello se explica por la alta incidencia que estos rubros tienen en la seguridad alimentaria y en la capacidad de desarrollo de las naciones, así como su posible impacto en la determinación del tipo de cambio. De allí que el conocimiento del comportamiento a futuro de dicha volatilidad sea vital para el diseño de políticas económicas y sociales. Los resultados muestran que, en general, históricamente los precios de los commodities tendieron a crecer hasta la década de los 2000s, para luego descender sin alcanzar los bajos niveles iniciales. La medición del nivel de volatilidad (desviaciones en torno a la media, con o sin corrección de tendencia), de los precios de los productos básicos de exportación, así como de índices de precios agropecuarios y de metales elaborados por el Fondo Monetario Internacional, la Standard \& Pool y el Bureau of Labor Statistics, con base en series originales y series suavizadas en diferencia y con promedio móvil centrado, apunta hacia mayores valores relativos en los productos minerales, siendo los resultados generalmente consistentes en los diferentes indicadores y enfoques empleados. La inclusión de diversos indicadores se hace buscando únicamente consistencia, por lo que no se destaca ninguno en particular. Probablemente, el único indicador que muestra resultados diferentes es el rango inter-anual de Parkinson, el cual sugiere una decreciente volatilidad de los grupos agropecuarios. Un análisis más a fondo sería conveniente, incorporando para ello algunos modelos estadísticos.

\section{Referencias}

Álvarez, M. \& Bermúdez, M. (2012). Evolución del comercio exterior de Colombia desde la óptica de los acuerdos comerciales. Ministerio de comercio, industria y turismo de la República de Colombia, documentos OEE-12.
ANIF (2016). Situación macro-financiera y fiscal de Colombia En: Colombia: Desarrollo empresarial y costos económicos de la paz. Seminario Macroeconómico y Feria de Servicios Empresariales, Bucaramanga.

Banco de la República (2014). Evolución de la balanza de pagos 2013. Bogotá.Balcombe, K. (2009). The nature and determinants of volatility in agricultural prices: an empirical study from 1962-2008. In: The evolving structure of world agricultural trade (Sarris A., Morrison J., eds), FAO, Italy, 109-136.

Bonifaz, J. \& Mortimore, M. (1999). Colombia: un CANalísis de su competitividad internacional. CEPAL, Serie Desarrollo Productivo 58.

CEPAL-FAO-IICA (2011). Volatilidad de precios en los mercados agrícolas (2000-2010). Implicaciones para América Latina y opciones de políticas. Perspectivas de la agricultura y del desarrollo rural en las Américas: Una mirada hacia América Latina y el Caribe. Boletín 1.

Departamento Administrativo Nacional de Estadística (DANE). Estadísticas de Comercio Internacional. Años varios. Disponibles en: http://www.dane.gov.co/

Echevarría, J. (1987). La evaluación de las exportaciones colombianas y sus determinantes. Un análisis empírico. Ensayos sobre política económica, 2: 257-294.Garay, L. (2004). Colombia: Estructura industrial e internacionalización 1967-1996. Biblioteca virtual del Banco de la República.

Gilbert, C. (2003). Trends and volatility in agricultural commodity prices. Ponencia presentada en State of Research and Future Directions in Agriculture Commodity Markets and Trade, FAO. Roma.

Gilbert, C. \& Morgan, C. (2010). Has food price volatility risen? Universitá degli Studi di Trento, Italia. Discussion paper 2/2010.

Huchet-Bourdon, M. (2011). Agricultural Commodity Price Volatility. OECD Food, Agriculture and Fisheries Papers, 52. OECD publishing. doi: http://dx.doi.org/10.1787/5kg0t00nrthc-en

Jacks, D., O'Rourke, K. \& Williamson, J. (2009). Commodity price volatility and world market integration since 1700 . NBER Working paper series 14748.

Jacks, D. S. (2013). From boom to bust: A typology of real commodity prices in the long run. NBER Working paper series 18874 .

Labys, W. (2006). Modeling and forecasting primery commodity prices. Ashgate publishing Ltd, 239pp.

Mesa, R., Gallón, \& Gómez, K. (2001). Entorno y evolución de las exportaciones colombianas en la década de los noventa. Lecturas de Economía, 55: 163-180.

Parkinson, M. (1980). The extreme value method for estimating the variance of the rate of return. Journal of Business, 53: 61-65.

Sumner, D. (2009). Recent commodity price movements in historical perspective. American Journal of Agricultural Economics, 91 (5): 1250-1256. 